\title{
Evanescent-mode-resonator-based and antenna-integrated wireless passive pressure sensors for harsh-environment applications
}

\author{
Haitao Cheng ${ }^{\mathrm{a},}$, Gang Shao $^{\mathrm{b}}$, Siamak Ebadi ${ }^{\mathrm{c}}$, Xinhua Ren ${ }^{\mathrm{d}}$, Kyle Harris ${ }^{\mathrm{e}}$, Jian Liu ${ }^{\mathrm{e}}$, Chengying Xu ${ }^{\mathrm{f}}$, Linan \\ $\mathrm{An}^{\mathrm{g}}$, and Xun Gong ${ }^{\mathrm{a}}$, \\ ${ }^{\text {a }}$ Antenna, RF and Microwave Integrated (AMRI) System Laboratory, Department of Electrical Engineering and Computer Science, University \\ of Central Florida, Orlando, FL 32816, USA \\ ${ }^{\mathrm{b}}$ School of Materials Science \& Engineering, Zhengzhou University, Zhengzhou, Henan 450001 China \\ ${ }^{\mathrm{c}}$ Intellectual Ventures, Bellevue, Washington 98005 U.S.A. \\ ${ }^{\mathrm{d}}$ Motorola Mobility Inc., Libertyville, IL 60048 U.S.A. \\ ${ }^{\mathrm{e}}$ Department of Mechanical and Aerospace Engineering, University of Central Florida, Orlando, FL 32816 USA \\ ${ }^{\mathrm{f}}$ Department of Mechanical Engineering, Florida State University, Tallahassee, FL 32310 USA \\ ${ }^{\mathrm{g}}$ Advanced Materials Processing and Analysis Center (AMPAC), University of Central Florida, Orlando, FL 32816 USA \\ *Corresponding author. Tel.: +1 858522 0178, E-mail: htcheng7@ gmail.com (H. Cheng) and Tel.: +1 407 823 5762, E-mail: \\ xun.gong@ucf.edu (X. Gong).
}

\begin{abstract}
A wireless pressure sensor for high-temperature applications is demonstrated based on a microwave evanescent-mode cavity resonator. Cavity deformation resulting from applied external pressure can be detected by measuring the resonant frequency change of the sensor. Compact sensor size is achieved by loading a cylindrical post inside the cavity resonator. In addition, a patch antenna is seamlessly integrated with the pressure sensor, without additional volume. This pressure sensor is able to survive high temperatures by adopting passive structures and robust ceramic/metallic materials. Fully-dense silicoaluminum carbonitride (SiAlCN) ceramic is used herein owing to its excellent thermal-mechanical properties and manufacturability as a Polymer-Derived Ceramic (PDC). A PDC soft-lithography technique is developed to fabricate the ceramic pressure sensor. In order to wirelessly interrogate the pressure sensor at high temperatures, a robust interrogation antenna is designed and fabricated with a wide fractional bandwidth. Finally, the cavity deformation of pressure sensor versus external pressure is measured at high temperatures up to $800^{\circ} \mathrm{C}$. The resonant frequency decreases from 11.75 to $11.56 \mathrm{GHz}$, when the applied external force on the sensor increases from 0 to 5 Newton at $800^{\circ} \mathrm{C}$.
\end{abstract}

Keywords: Evanescent-mode resonator, harsh environment, integrated antenna, Polymer-Derived Ceramic, pressure sensor, wireless passive sensor.

\section{Introduction}

Real-time pressure monitoring is essential for many harsh-environment applications, such as gas turbines for power generation. During turbine operations, there is an optimal pressure ratio to achieve the maximal thermal efficiency for a given temperature [1]. Pressures beyond or below the optimal value will result in a reduced thermal efficiency. Additionally, online monitoring of pressure inside a gas turbine is necessary for safe operations. Higher pressure ratios than the designed value could lead to compressor surge which results in flameout, or even serious damage to compressor blades.

Therefore, in order to improve thermal efficiency and guarantee operational safety of gas turbines, pressure sensors are highly desirable inside combustion chambers. These sensors are required to reliably work in the harsh environment consisting of high temperatures $\left(1000-1400^{\circ} \mathrm{C}\right)$, high pressures (300-600 psi), and corrosive gases (containing sodium, vanadium and sulfate). Pressure sensors with passive, wireless, and robust characteristics are highly desirable to survive this harsh environment.

For aforementioned applications, pressure sensors need to be passive due to the fact that semiconductor devices and circuits typically fail at temperatures above $600^{\circ} \mathrm{C}[2,3]$. Passive pressure sensors based on resistive [4, 5] or capacitive [6, 7] sensing mechanisms were proposed to survive high temperatures above $400^{\circ} \mathrm{C}$. However, wire interconnection is required to interrogate these sensors, which is main failure mechanism for gas turbine applications [8]. By using capacitive sensing mechanism and inductive coupling, pressure sensors were demonstrated wirelessly for high-temperature applications [9-11]. However, quality (Q) factors of $L C$ resonators are usually very limited, especially at high temperatures. For example, $\mathrm{Q}$ factor of the pressure sensor in [9] is only 6 at $400^{\circ} \mathrm{C}$. Alternatively, evanescent-mode resonators, with high $\mathrm{Q}$ factors, were utilized to wirelessly detect pressure [12], air flow [13] and temperature [14] by measuring cavity deformations. In these sensors [12, 13], antennas were designed separately therefore adding additional volume to the entire sensor structure. In addition, the aforementioned evanescent-mode sensors operate below $90^{\circ} \mathrm{C}$. In this paper, we will demonstrate a compact wireless passive pressure sensor for harsh-environment applications using high-Q evanescent-mode resonators, robust ceramic and metallic materials, and integrated antenna. 
The authors presented an evanescent-mode-resonator-based wireless passive pressure sensor design with integrated slot antenna to achieve compact size [15]. In this paper, we will use a similar evanescent-mode resonator structure [15] but employ an integrated patch antenna to enhance the sensing range. This integrated patch antenna also exhibits zero additional volume. The small size of the pressure sensor can minimize the adverse effects on the aerodynamic flow inside turbines or other devices. The proposed pressure sensor is made of high-temperature-stable materials including fully-dense SiAlCN and platinum, which have been proven to work reliably above $1000^{\circ} \mathrm{C}$ [16-18]. This pressure sensor will be wirelessly interrogated by a high-temperaturestable antenna without the need of wire connections to the sensor.

The schematic of the pressure sensor structure and wireless interrogation mechanism is illustrated in Fig. 1. The resonant frequency $f_{r}$ of the evanescent-mode resonator is determined by [19]:

$$
f_{r}=\frac{1}{2 \pi \sqrt{L\left(c_{p}+c_{r}\right)}}
$$

where $c_{p}$ represents parallel-plate capacitance between the sensor cap and the cylindrical post inside the cavity, $c_{r}$ is the remaining fringing capacitance, and $L$ is the equivalent inductance. $c_{p}$ can be approximately given by:

$$
c_{p}=\frac{\varepsilon_{0} A}{g a p}
$$

where the top surface of the cylindrical post has an area of $A$, and gap is the distance between the sensor cap and the post. With pressure being applied on the cavity, $c_{p}$ increases with the reduced gap due to cavity deformation. Therefore, the pressure can be wirelessly monitored by measuring the change in $f_{r}$. By adding the post structure in the cavity resonator as shown in Fig. 1, high sensitivity of the pressure senor is achieved due to a small gap value [20]. It is worthy of mentioning that this type of evanescentmode resonators have been also used for high-Q widely-tunable filters [21-24], owing to the merits of reduced size and high sensitivity.

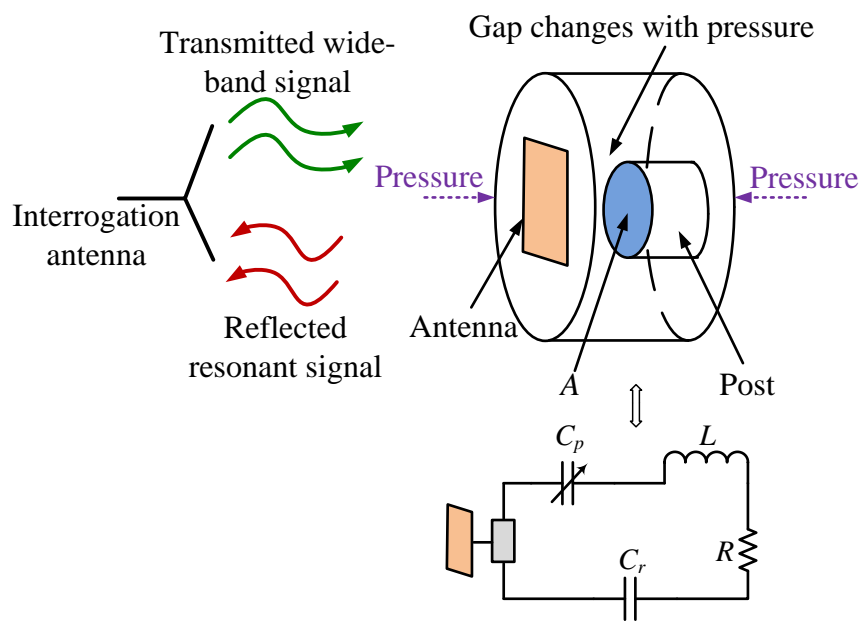

Fig. 1. Schematic and circuit model of the wireless pressure sensor based on an evanescent-mode cavity resonator.

\section{Characterization of the fully-dense SiAICN PDC}

Structure material of the pressure sensor is polymer-derived ceramic which exhibits excellent thermal-mechanical properties at high temperatures $[16,17]$. However, as a novel ceramic material, the dielectric properties at microwave frequency including dielectric constant and loss tangent were still unknown. Therefore, it is necessary to measure the dielectric properties of the fullydense SiAlCN PDC before the sensor design and fabrication. Dielectric characterization is carried out by using the microwave cavity method presented in [18]. 


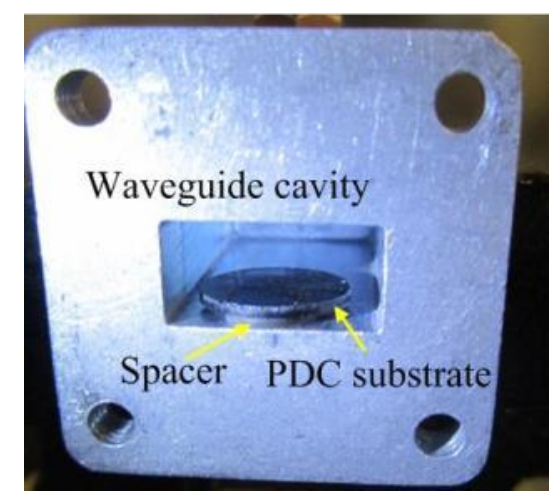

Fig. 2. Characterization of dielectric properties of the SiAlCN PDC in a microwave cavity at room temperature.

The fully-dense SiAlCN PDCs are synthesized by using $10 \%$ and $30 \%$ poly (melamine-co-formaldehyde) acrylated solution (PVN, Sigma-Aldrich, St. Louis, MO) [25]. The SiAlCN PDC substrate is placed on a spacer inside a $K_{u}$-band waveguide cavity for dielectric characterization, as shown in Fig. 2. Then the dielectric constant and loss tangent of the SiAlCN PDC can be extracted from the resonant frequency and loaded Q factor of the waveguide cavity [18]. The resonant frequencies (loaded Q factors) for PDCs with $10 \%$ and $30 \% \mathrm{PVN}$ are $9.793 \mathrm{GHz}(1924)$ and $9.806 \mathrm{GHz}$ (1299), respectively. The dielectric constant (loss tangent) for PDCs with 10\% and 30\% PVN are found to be $5.1(0.01)$ and $6.3(0.05)$, respectively. SiAlCN PDC with $10 \%$ PVN is selected to fabricate the sensor cap due to its lower dielectric loss. However, SiAlCN PDC with 30\% PVN is used for sensor bottom since this composition can provide thick substrates without warping. Since the sensor bottom will be completely metallized using platinum, the loss inside the lossy PDC with 30\% PVN will not affect the Q factor the pressure sensor.

\section{Design of the pressure sensor and robust interrogation antenna}

This wireless pressure sensor is designed for high-temperature applications, and therefore constraints from microwave resonator design, ceramic synthesis and fabrication limitation need to be carefully considered throughout the sensor design. A patch antenna is seamlessly integrated with the pressure sensor to receive the interrogation signal and transmit the sensor response. In addition, a robust interrogation antenna with sufficiently-wide fractional bandwidth is developed in order to perform sensor measurements.

\subsection{Design of the pressure sensor based on an evanescent-mode cavity resonator}

The resonant frequency can be reduced by increasing sensor diameter and decreasing gap size. The $\mathrm{Q}$ factor will increase for growing gap size [26]. The considerations from microwave resonator design, ceramic synthesis and machining are listed as follows. (1) There is a tradeoff between sensor size and Q factor. When the resonator is heavily loaded (for a small sensor gap), the resonant frequency is reduced due to the large $c_{p}$ as shown in equation (2). The reduced resonant frequency will lead to less free-space path loss and therefore increase sensing range. However, the associated reduction in Q factor will adversely affect the sensing range. Therefore, iterations in design are needed to maximize the sensing range. (2) The thickness of fully-dense SiAlCN PDC is limited to be below $1500 \mu \mathrm{m}$ in order to avoid cracks or warping during sintering. Details will be provided in Section 4.1. (3) The pressure sensor needs to be able to respond to 0-600 psi pressure change without mechanical structure breakdown. If the cap of the sensor is too thick, the pressure sensor is not sensitive to the pressure change. However, cap thicknesses below a critical value will lead to RF shorting when the cap touches the post. In addition, the sensor cap may break down when the stress caused by the external pressure exceeds the ultimate tensile strength (UTS) of the PDC ceramic, which is approximately 1000 MPa. 


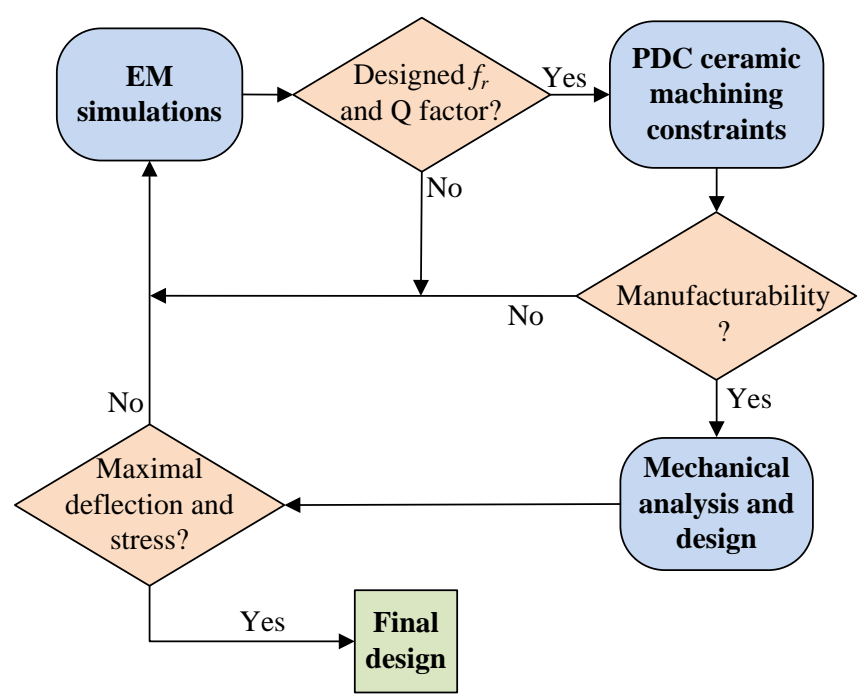

Fig. 3. Flow chart to design the microwave pressure sensor.

The design flow chart is presented in Fig. 3. Sensor designs remain in the design loop until all these three requirements are met. It is noted that in order to leave design margin to prevent RF shorting between the cap and post due to the overly-estimated UTS or fabrication tolerances, the evanescent-mode sensor is designed with medium-level loading. Therefore, the frequency downshift effect is not as drastic as in the highly-loaded resonators. Optimization of Q values becomes the most important to maximize the sensing distance and accuracy. The frequency reduction is mainly achieved by increasing the sensor lateral dimensions, which are ultimately limited by the material manufacturability. The dimensions of the pressure sensor after optimization are listed in Fig. 4(a). The relationship between resonant frequency and gap size is presented in Fig. 4(b) using ANSYS High Frequency Structure Simulator (HFSS) simulations. It is noted that the resonant frequency decreases when the gap size (external pressure) becomes smaller (larger). The sensitivity of the sensor becomes higher when the gap size is smaller.

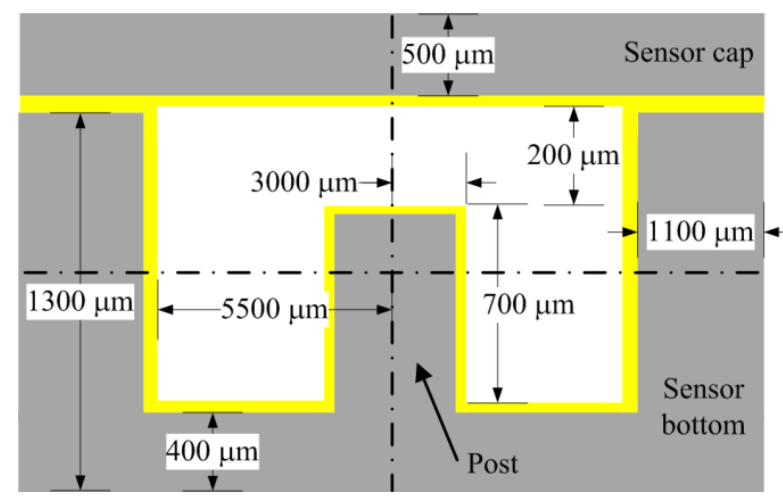

(a)

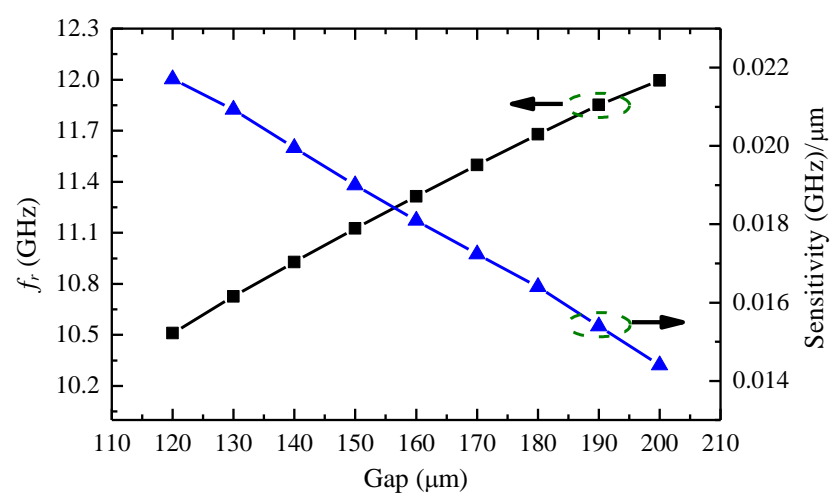

(b)

Fig. 4. The evanescent-mode-resonator pressure sensor after design. (a) Side view with dimensions. (b) Simulated resonant frequency of the pressure sensor versus cavity gap based on Fig. 4(a).

To verify the robustness under pressure, this pressure sensor was simulated in ANSYS Mechanical. As shown in Fig. 5(a), the deflection of the sensor cap is found to be $69.8 \mu \mathrm{m}$ when the external pressure is $600 \mathrm{psi}$, which makes sure that no RF shorting will occur during sensor operation. In addition, the maximal stress in the sensor cap is simulated to be $298.6 \mathrm{MPa}$ as shown in Fig. 5(b), which is much smaller than the UTS of PDC. During this mechanical simulation, the Young's modulus of the SiAlCN PDC is set as $100 \mathrm{GPa}$, and the bottom of the sensor is assumed to be flat, which corresponds to the case when the pressure sensor is mounted on a rigid surface. The sensor sensitivity can be calculated as $2.44 \mathrm{MHz} / \mathrm{psi}$, by multiplying the frequency-defection sensitivity shown in Fig. 4(b) with the defection-pressure sensitivity shown in Fig. 5(a). 


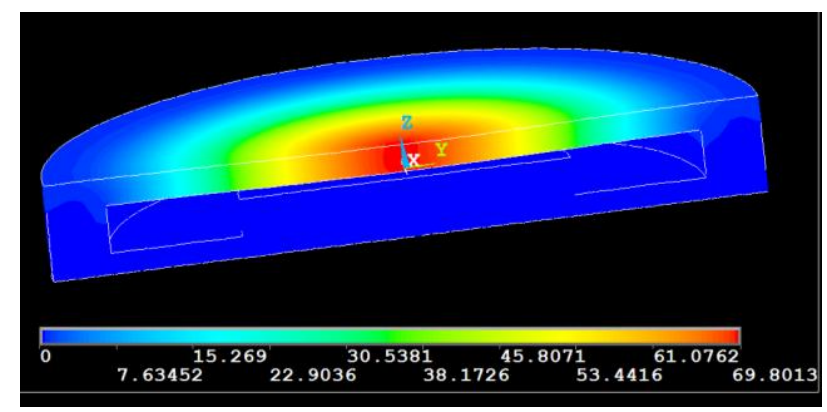

(a)

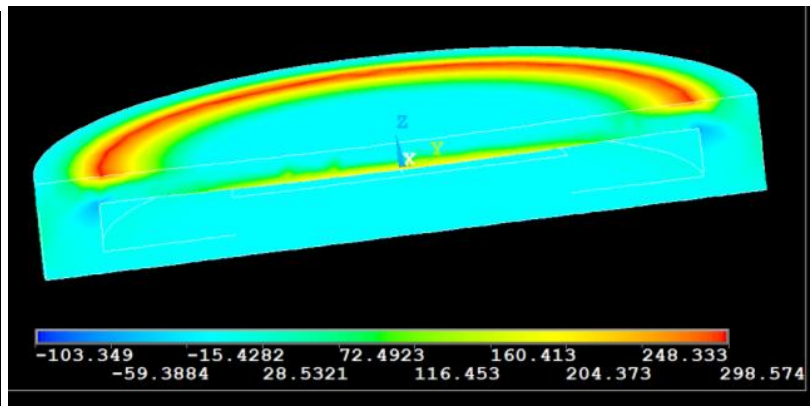

(b)

Fig. 5. Mechanical simulations of the pressure sensor under 600 psi pressure. (a) Sensor deformation (in $\mu$ m). (b) Stress distribution (in GPa).

\subsection{Design of the patch antenna coupled with the pressure sensor}

A patch antenna is designed to be seamlessly integrated with the pressure sensor in order to facilitate the wireless interrogation and minimize size as shown in Fig. 6. The patch antenna is located on the top surface of the sensor cap and coupled to the pressure sensor though a rectangular slot etched inside the ground plane on the bottom of the sensor cap. Similar to the filter/antenna integration technique presented in [27], this integrated patch antenna occupies zero additional volume and exhibits close to $100 \%$ antenna efficiency.

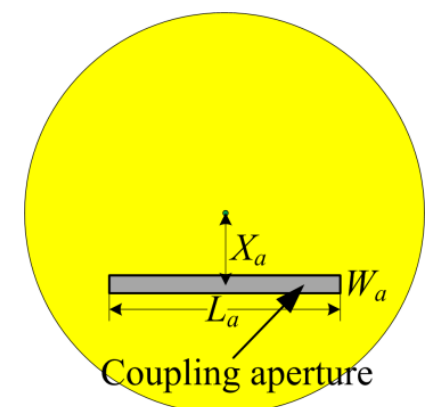

(a)

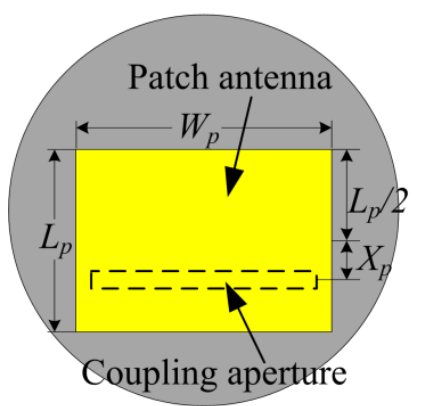

(b)

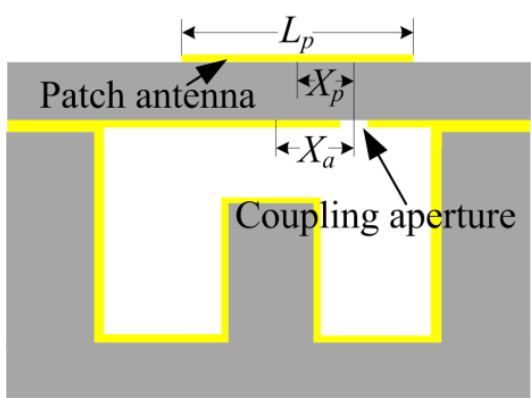

(c)

Fig. 6. Schematic of the patch antenna integrated with the pressure sensor. Bottom (a) and top (b) view of the sensor cap. (c) cut view of the sensor and patch antenna.

The patch antenna is designed using the dielectric properties measured in Section 2. The patch length $L_{p}$ and width $W_{p}$ are found to be 5.4 and $7.1 \mathrm{~mm}$, respectively at the resonant frequency of the pressure sensor, i.e. $f_{r}=11.9 \mathrm{GHz}$. It is noted that $\varepsilon_{r}=$ 5.36 (assuming 5\% increase from $\varepsilon_{r}=5.1$ at room temperature) is used for the PDC with $10 \% \mathrm{PVN}$ at $700^{\circ} \mathrm{C}$, based on the temperature-dependent dielectric properties of similar SiCN ceramics reported in [18].

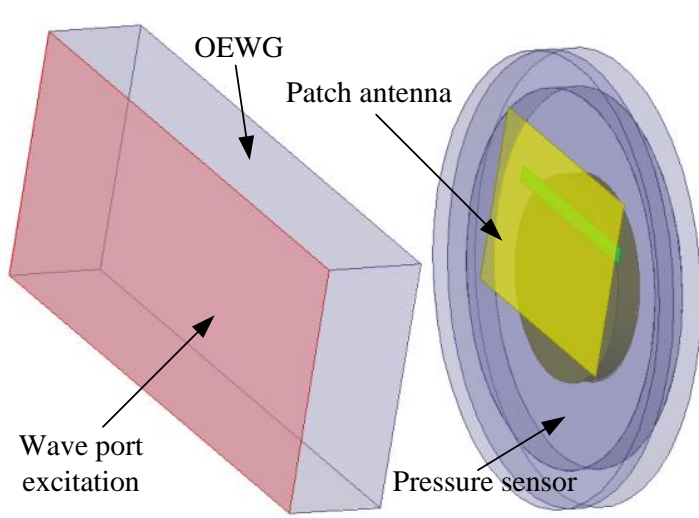

(a)

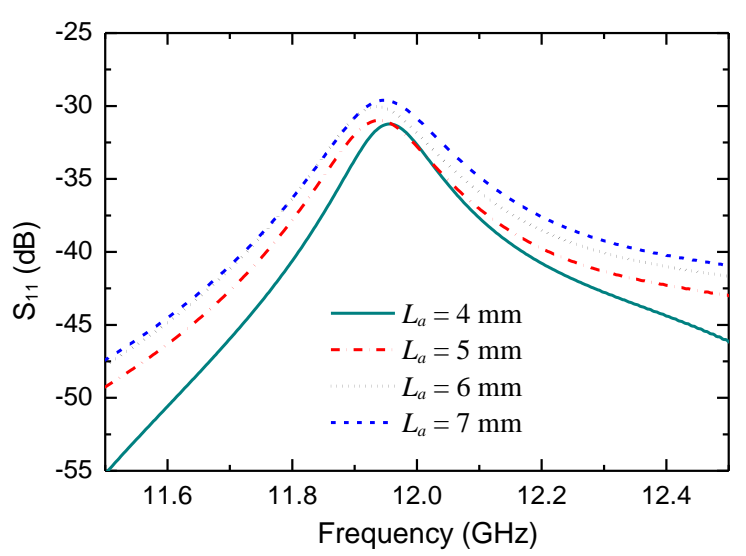

(b) 


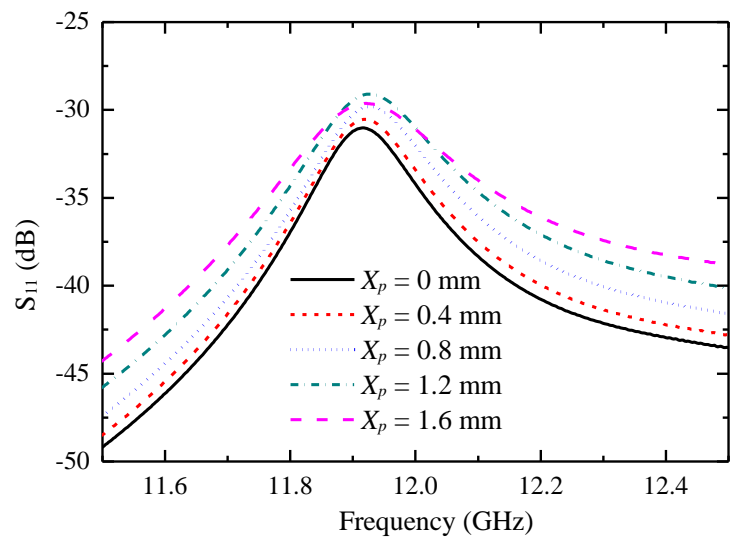

(c)

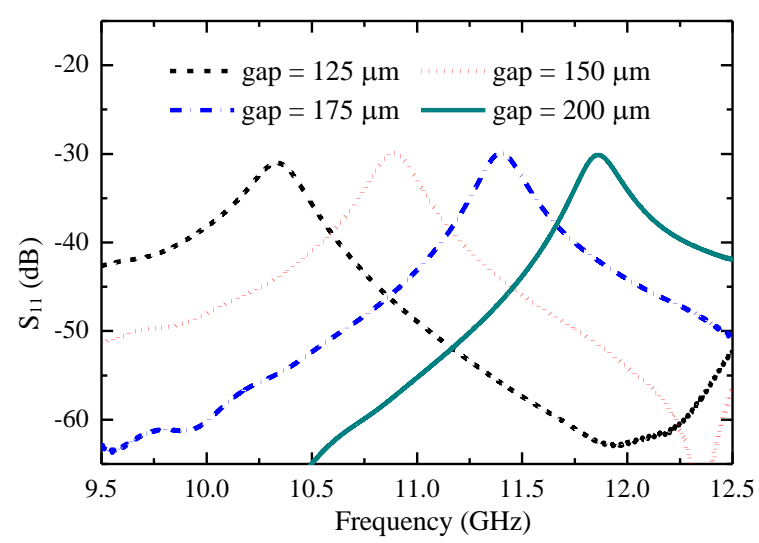

(d)

Fig. 7. Design of the patch antenna. (a) Simulation setup for the wireless interrogation of the pressure sensor in HFSS. Parametric study of (b) patch length $L_{a} . X_{p}=0.8 \mathrm{~mm}$ is used. (c) Coupling position $X_{p}$ for the integrated patch antenna. $L_{a}=6 \mathrm{~mm}$ is used. (d) Sensor responses versus different gap sizes inside the cavity.

The length of the coupling aperture $L_{a}$ and location of the patch antenna $X_{p}$ are then studied and optimized for maximum sensing range in HFSS. In order to carry out this parametric study, an $X$-band open-ended waveguide (OEWG) antenna with an aperture size of $22.86 \times 10.16 \mathrm{~mm}^{2}$ is used to interrogate the pressure sensor as shown in Fig. 7(a). The dimensions of the pressure sensor can be found in Fig. 4(a). This OEWG antenna transmits a wide-band RF signal, which couples to the sensor through the integrated patch antenna. Only the frequency components close to the resonant frequency of the sensor resonate for many cycles and re-radiate back to the OEWG. By using a time-domain gating technique [28], the responses corresponding to the resonance inside the pressure sensor are isolated and identified by the network analyzer. OEWG antenna $S_{11}$ versus $L_{a}$ and $X_{p}$ are plotted in Fig. 7(b) and Fig. 7(c), respectively. The peaks of the curves correspond to the resonant frequency of the pressure sensor. Finally, $L_{a}=6 \mathrm{~mm}$ and $X_{p}=0.8 \mathrm{~mm}$ are chosen to achieve a good compromise between the received signal strength and Q factor (which determines the sharpness of the peaks). During the antenna design, the width and location of the coupling aperture are fixed as $W_{a}=0.5 \mathrm{~mm}$ and $X_{a}=2 \mathrm{~mm}$, respectively, to simplify the antenna design. The dimensions of the patch antenna and coupling aperture are summarized in Table 1 . To verify that this wireless interrogation can detect the resonant frequency change of the sensor, simulated sensor responses versus different gap sizes are shown in Fig. 7(d). It is apparent that under different external pressures, the resonant frequency of the pressure sensor can be wirelessly detected.

Table 1. The dimensions of the patch antenna.

\begin{tabular}{ccccccc}
\hline Symbol & $W_{p}$ & $L_{p}$ & $W_{a}$ & $L_{a}$ & $X_{a}$ & $X_{p}$ \\
\hline Parameters & Patch & Patch & Coupling & Coupling & Coupling aperture with & Patch location with \\
& width & length & aperture width & aperture length & respect to the cavity center & respect to the aperture \\
Values $(\mathrm{mm})$ & 7.1 & 5.4 & 0.5 & 6 & 2 & 0.8 \\
\hline
\end{tabular}

\subsection{Design of the robust interrogation antenna with wide bandwidth.}

Ideally, the wireless pressure sensor needs to be tested within a chamber with both high temperatures (up to $1000^{\circ} \mathrm{C}$ ) and high pressures (up to $600 \mathrm{psi}$ ). In addition, RF signals need to be sent to the inside of the chamber in order to wireless interrogate the sensor. All these requirements call for very complicated and challenging measurement setup. In this paper, we will focus on the demonstration of the working mechanism of this novel wireless passive pressure sensors. Therefore, a measurement setup as shown in Fig. 8(a) is used instead. The temperature of the sensor is controlled by a heat pad under the pressure sensor. While the pressure on the sensor is applied with an alumina rod passing through the interrogator antenna. This simple setup allows us to emulate the sensor cavity deformation caused by ambient pressure change. It should be noted that the loading effect of the alumina rod on the resonant frequency of the pressure sensor is negligible, i.e. a slight frequency down shift of $0.0048 \mathrm{GHz}$ at $11.9 \mathrm{GHz}$ through HFSS simulations. 


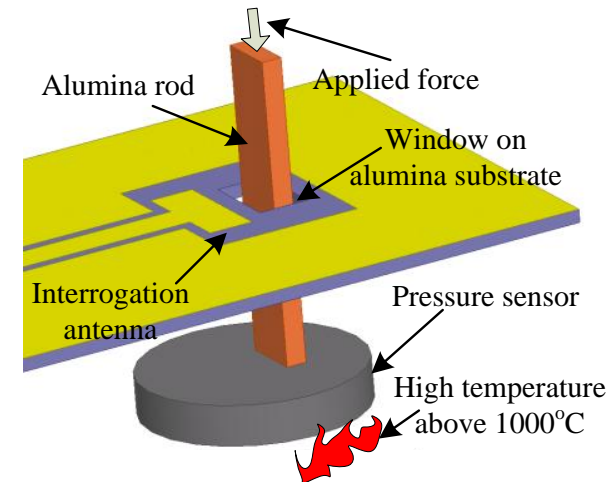

(a)
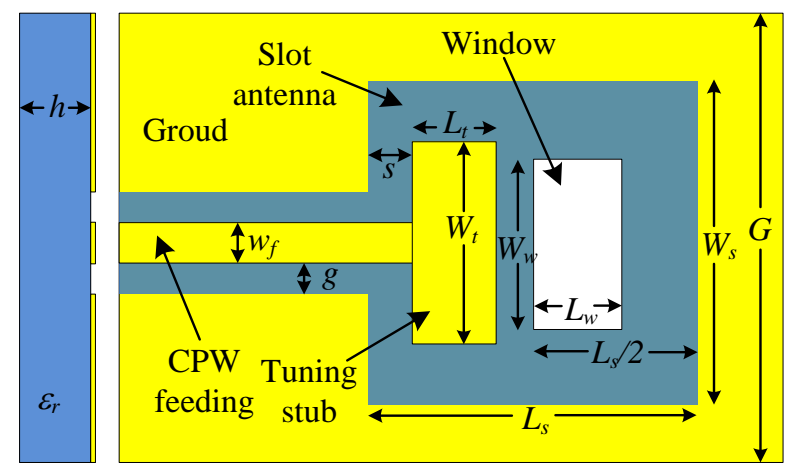

(b)

Fig. 8. Interrogation antenna for pressure sensor measurement at high temperatures. (a) The schematic of the pressure sensor measurement with the interrogation antenna. (b) The schematic of the slot antenna loaded with a rectangular tuning stub.

There are two requirements for the interrogation antenna: (1) it needs to be robust to survive high temperatures; (2) its fractional bandwidth should be wide enough to cover the entire frequency range of the pressure sensor for different pressures. A robust antenna to interrogate temperature sensor was proposed in [29], and it is able to survive high temperatures up to $1300^{\circ} \mathrm{C}$ by using an alumina substrate and a platinum metal layer. Similarly, a robust antenna is designed herein for the pressure sensor measurement. As shown in Fig. 8(b), a tuning stub with CPW feeding is loaded inside the slot antenna to improve the antenna bandwidth. A window is cut inside the antenna substrate, allowing the alumina rod to apply a force on the pressure sensor.

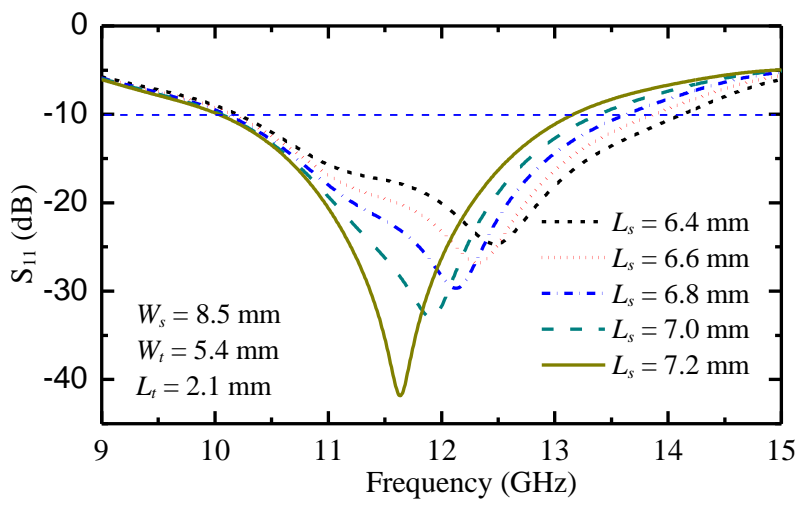

(a)

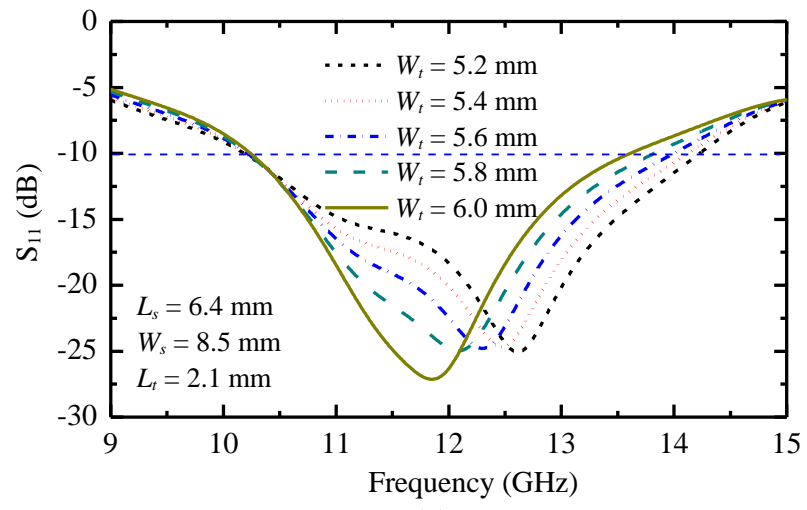

(c)

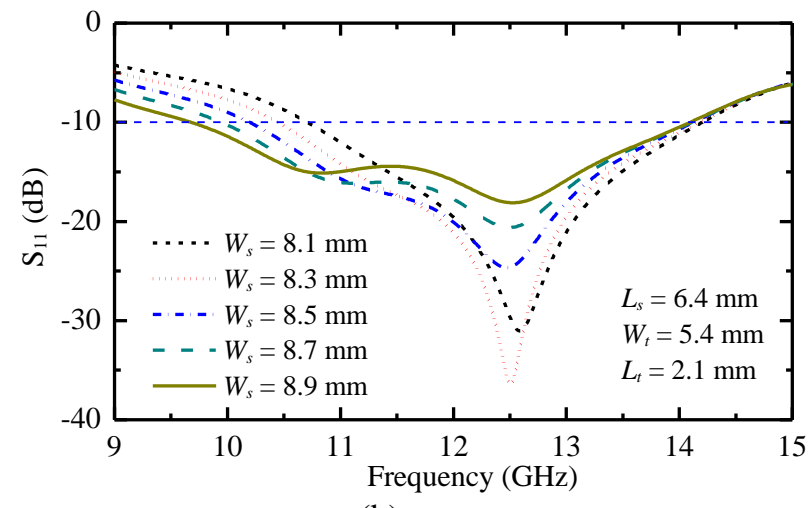

(b)

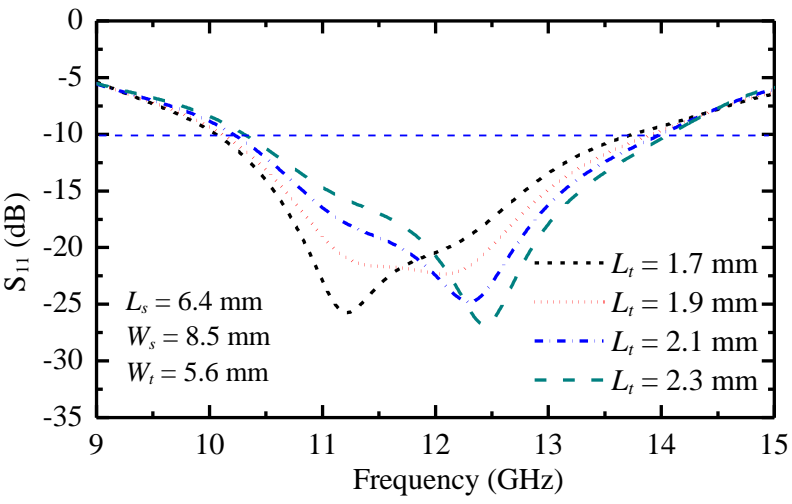

(d)

Fig. 9. Parameter study of (a) the slot length $L_{s}$, (b) the slot width $W_{s}$, (c) the tuning-stub width $W_{t}$ and (d) the tuning-stub length $L_{t}$ of the interrogation antenna.

As shown in Fig. 9, the slot length $L_{s}$, slot width $W_{s}$, tuning-stub length $L_{t}$, and tuning-stub width $W_{t}$ are studied to optimize the antenna bandwidth. It is noted that the spacing between the tuning stub and the slot edge is chosen as $s=0.6 \mathrm{~mm}$. Fig. 9 (a) shows that the upper bound of the antenna impedance matching bandwidth is increased for smaller $L_{s}$. So $L_{s}=6.4$ mm is chosen for this antenna design. Then it is observed in Fig. 9(b) that the lower bound of the antenna impedance matching bandwidth is determined by $W_{s}$. Larger $W_{s}$ leads to a wider antenna bandwidth but worse impedance matching. Therefore, $W_{s}=8.5 \mathrm{~mm}$ is used as the tradeoff between antenna bandwidth and impedance matching. Finally, the effects of $W_{t}$ and $L_{t}$ are studied and shown in Fig. 9(c) and Fig. 9(d), respectively. $W_{t}=5.6 \mathrm{~mm}$ and $L_{t}=2.1 \mathrm{~mm}$ are chosen for better impedance matching. The dimensions of 
the designed interrogation antenna are summarized in Table 2. The designed interrogation antenna exhibits a fractional bandwidth of $31 \%\left(\mathrm{~S}_{11}<-10 \mathrm{~dB}\right)$ at the center frequency of $12.1 \mathrm{GHz}$.

Table 2. The dimensions of the interrogation antenna.

\begin{tabular}{ccc}
\hline Symbol & Parameters & Values $(\mathrm{mm})$ \\
\hline$w_{f}$ & Feed width & 2.5 \\
$g$ & Gap & 0.63 \\
$s$ & Spacing & 0.6 \\
$W_{t}$ & Tuning-stub width & 5.6 \\
$L_{t}$ & Tuning-stub length & 2.1 \\
$W_{s}$ & Slot width & 8.5 \\
$L_{s}$ & Slot width & 6.4 \\
$G$ & Ground size & 22.9 \\
$h$ & Substrate thickness & 0.635 \\
$L_{w}$ & Window length & 2.5 \\
$W_{w}$ & Window width & 4 \\
\hline
\end{tabular}

As shown in Fig. 8(a), an alumina rod with a cross- sectional area of $0.9 \times 0.6 \mathrm{~mm}^{2}$ is inserted though the interrogation antenna. The loading effect of the alumina rod on resonant frequency of the interrogation antenna is also verified in HFSS simulations. It is observed that the center frequency of the interrogation antenna is decreased by $0.1 \mathrm{GHz}$ while the antenna fractional bandwidth remains the same, which is still sufficient to cover the sensor responses.

\section{Fabrication of the PDC pressure sensor and the robust interrogation antenna}

In this section, the details of sensor fabrication using fully-dense PDC and platinum, as well as the interrogation antenna fabrication are presented.

\subsection{Fabrication process of PDC pressure sensor}

Commercially available preceramic precursor of polysilazane (HTT1800, Kion, Huntingdon Valley, PA) was used as the PDC precursor. Aluminumtri-sec-butoxide (ASB, Sigma-Aldrich, St. Louis, MO) and poly (melamine-co-formaldehyde) acrylated solution (PVN, Sigma-Aldrich, St. Louis, MO) were used as Al and N sources, respectively. Bis (2, 4, 6trimethylbenzoyl)-phenylphosphineoxide (Irgacure 819, CibaSpecialty Chemicals Inc., Basal, Switzerland) was used as the photoinitiator. The detailed synthesis process was reported in [25].

PDCs with thickness above $500 \mu \mathrm{m}$ typically exhibit cracks or warping [30, 31]. To avoid this issue, a new PDC softlithography fabrication method is developed to realize PDC substrates up to 1500 - $\mu$ m thick without the aforementioned problems.

The fabrication process is outlined in Fig. 10 and described as follows. (1) Teflon was chosen as the mould material due to its non-stick and easy-to-machine properties. As shown in Fig. 10(a), a Teflon sheet was machined by micromilling using a three-axis machine tool with motion accuracy of $0.25 \mu \mathrm{m}$ [32]. In order to compensate for the $31 \%$ dimensional shrinkage during ceramic sintering, the Teflon mould dimensions were proportionally enlarged in machining. For easier demoulding, silicon spray lubricant (3-IN-ONE 10041) was applied on the Teflon mould before the soft-lithography process. (2) The PDC precursor in liquid phase was filled into the Teflon mould as shown in Fig. 10(b). A quartz glass was then used to cover the top of the PDC precursor. Similarly, to avoid adhesion, a thin layer of Teflon film was attached to the quartz glass. (3) As shown in Fig. 10(c), the PDC precursor was exposed to UV light (Newport 66923) for 10 minutes at ambient atmosphere environment. After the lithography, the liquid ceramic precursor consisting of photo initiator was transformed into solid polymer. Then the polymer was removed from the Teflon mould, as shown in Fig. 10(d). (4) Pre-pyrolysis was carried out at $150^{\circ} \mathrm{C}$ for twenty-four hours in order to remove the residual stress which was generated during the fabrication process of molding and cross-linking of the polymer. After that, the polymer was pyrolyzed at $1000^{\circ} \mathrm{C}$ for four hours and converted to a fully-dense SiAlCN ceramic, as shown in Fig. 10(e). (5) Fig. 10(f) shows the pressure sensor bottom before and after PDC sintering. Similarly, the sensor cap was fabricated with a thickness of $490 \mu \mathrm{m}$ which is smaller than the design value of $500 \mu \mathrm{m}$ due to fabrication tolerance. 


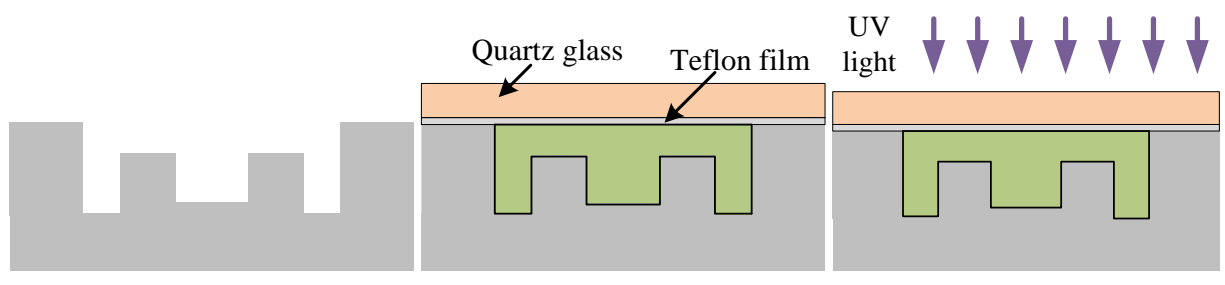

(a)

(b)

(c)

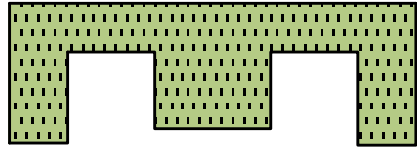

(d)

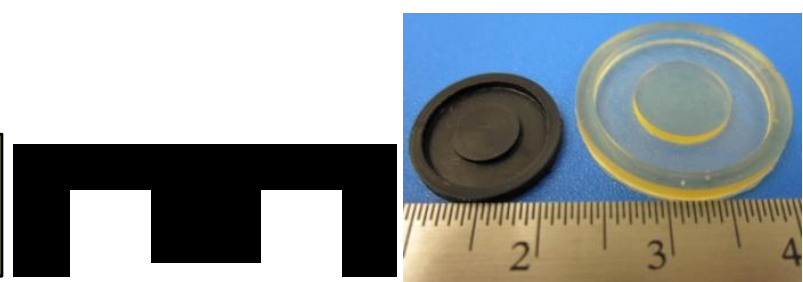

(e)

(f)

Fig. 10. Fabrication process flow of the PDC sensor bottom. (a) Machining of the Teflon mould. (b) Liquid ceramic precursor in the mould. (c) UV exposure. (d) Polymer demoulding. (e) PDC sintering. (f) Photos of the polymer (right) and sintered PDC (left).

After the PDC machining, a thin layer of platinum was metalized on the PDC sensor bottom, which is necessary to form a microwave cavity resonator. This platinum metallization process was also used to fabricate robust antennas [29] and temperature sensors [33]. Platinum paste (ESL 5542) was applied to the PDC surface, and then dried for 2 minutes at $110^{\circ} \mathrm{C}$. After that, the platinum paste was sintered at $1000^{\circ} \mathrm{C}$ for 10 minutes with a ramping rate of $10^{\circ} \mathrm{C} / \mathrm{min}$. The platinum paste was repeatedly applied for three times to form a thickness of approximately $25 \mu \mathrm{m}$ without voids. Fig. 11(a) shows the sensor bottom after the platinum metallization. A 3-D profile of the sensor bottom was measured by using a digital microscope (KEYENCE VHX-1000) and shown in Fig. 11(b). The dimensions of the metallized cavity are summarized in Fig. 11(c).

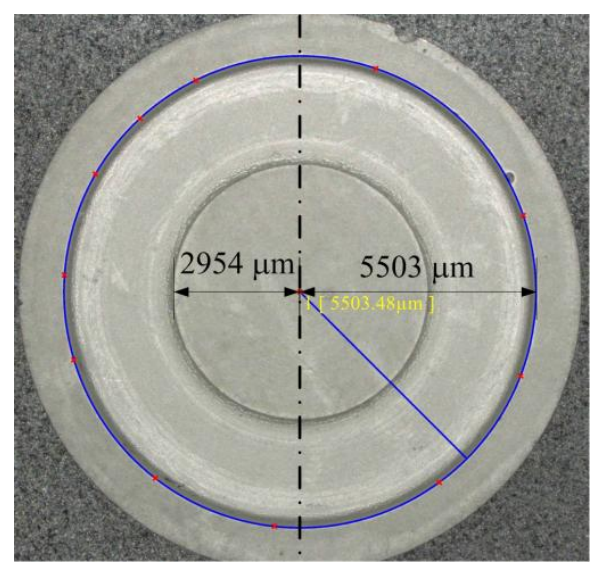

(a)

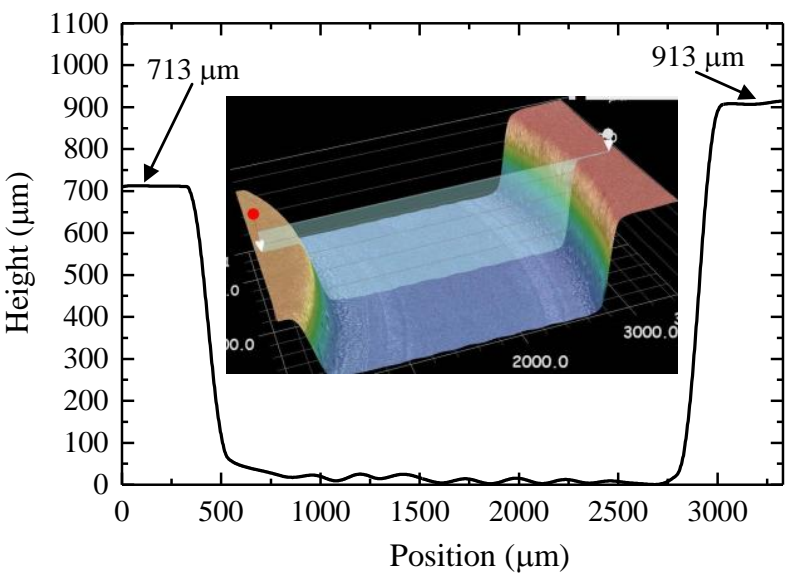

(b)

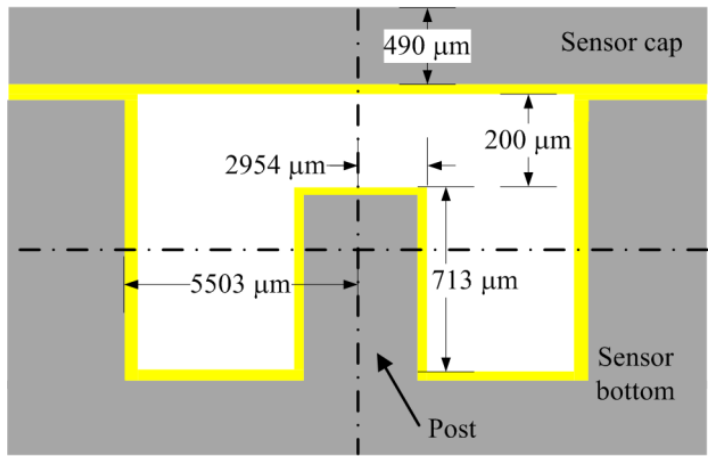

(c)

Fig. 11. Fabricated PDC cavities. (a) Top view and (b) 3-D profile of the PDC sensor bottom after the platinum metallization. (c) Cavity dimensions after fabrication. 
In the next step, platinum is patterned on both bottom and top surfaces of PDC sensor cap. As shown in Fig. 12(a), the PDC bottom is uniformly metalized to form the ground plane of patch antenna, except for the rectangular coupling aperture. In addition, the rectangular patch antenna is patterned and shown in Fig. 12(b). It should be noted that this platinum pattern process is also used to fabricate the robust interrogation antenna, which will be discussed in the Section 4.2. At last, the PDC sensor bottom and cap are bonded with a thin layer of platinum paste after a high-temperature sintering process and shown in Fig. 12(c).

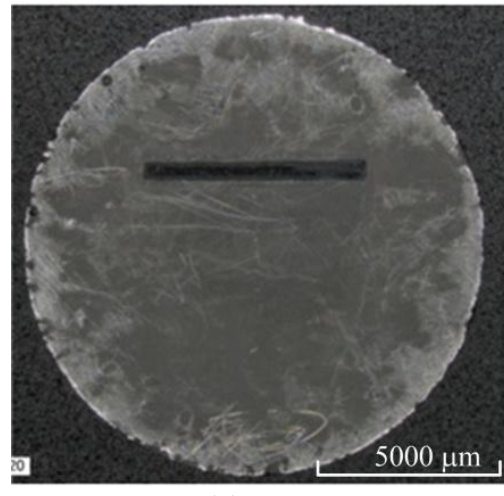

(a)

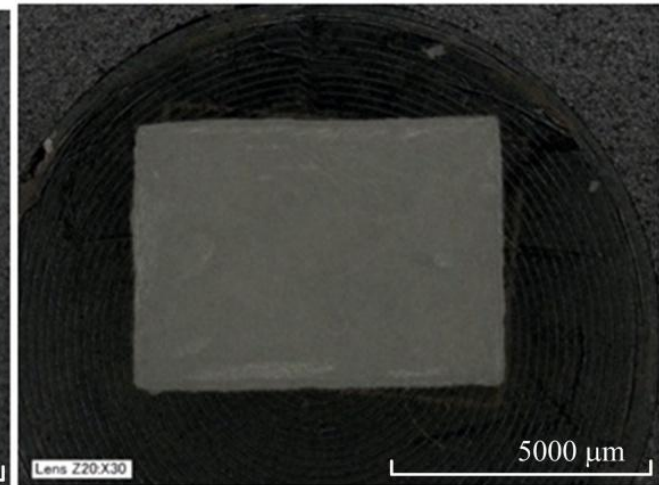

(b)

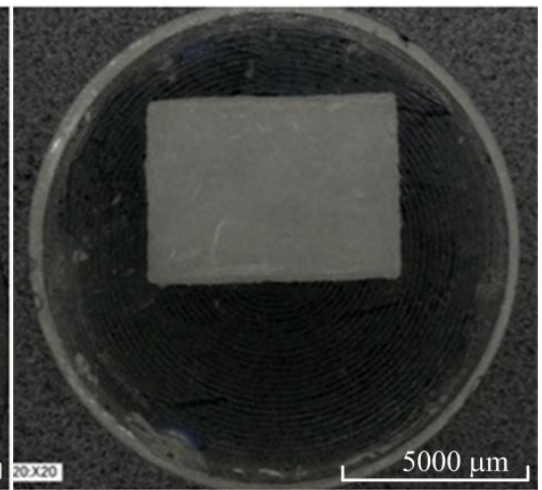

(c)

Fig. 12. Platinum metallization and bonding process. (a) The coupling aperture on the ground plane of the patch antenna. (b) The patch antenna. (c) The bonded pressure sensor consisting of both PDC sensor bottom and cap.

\subsection{Fabrication of the robust interrogation antenna}

The robust interrogation antenna was fabricated by using alumina substrate and platinum layer in order to survive high temperatures. The fabrication process to pattern the platinum film was specially developed and shown in Fig. 13. (1) The antenna layout was printed on a transfer paper by using a laser printer with a printing resolution of 1200 dpi as shown in Fig. 13(a). (2) A window with dimensions of $2.5 \times 4 \mathrm{~mm}^{2}$ was cut on an alumina substrate by using laser machining (Laserage Technology Corporation) as shown in Fig. 13(b). (3) The transfer paper was taped on the alumina substrate, while the antenna layout was aligned with the window on the alumina substrate. After a thermal compression process by using a thermal laminator, the antenna layout on the transfer paper was transferred to the alumina substrate as shown in Fig. 13(c). (4) Platinum paste (ESL 5542) was applied based on the transferred antenna layout under a microscope. Then the paste was dried by using a hotplate for 2 minutes at $110^{\circ} \mathrm{C}$ as shown in Fig. 13(d). (5) Fig. 13(e) shows the antenna after the high-temperature sintering process for platinum.

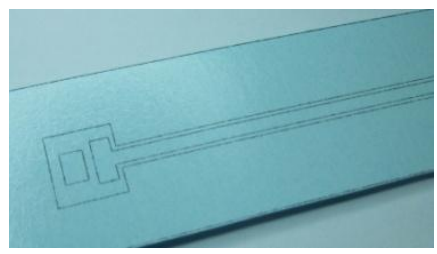

(a)

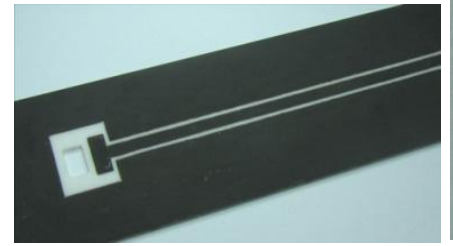

(d)

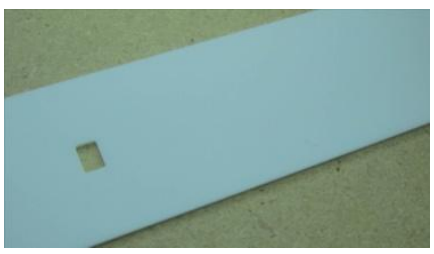

(b)

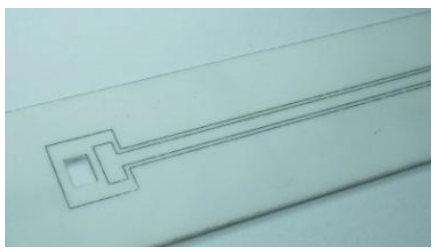

(c)

Fig. 13. Fabrication of the robust interrogation antenna. (a) Antenna layout on a transfer paper. (b) Window cuting on an alumina substrate. (c) Antenna layout transered to the alumina substrate. (d) Pattern of platinum paste. (e) Platinum pattern after high temperature sintering.

In order to measure the interrogation antenna, Thru-Reflect-Line (TRL) calibration was used to provide accurate antenna characterization at the reference plane as shown in Fig. 14 [34]. The measured fractional bandwidth of $31 \%$ and center frequency of $11.8 \mathrm{GHz}$ closely match the simulated bandwidth of $31 \%$ and center frequency of $12.1 \mathrm{GHz}$, as shown in Fig. 14. The fractional bandwidth and center frequency are the two most important factors for sensor interrogation. The measured $S_{11}$ has 
multiple ripples in the antenna bandwidth due to impedance discontinuity in the long CPW line.

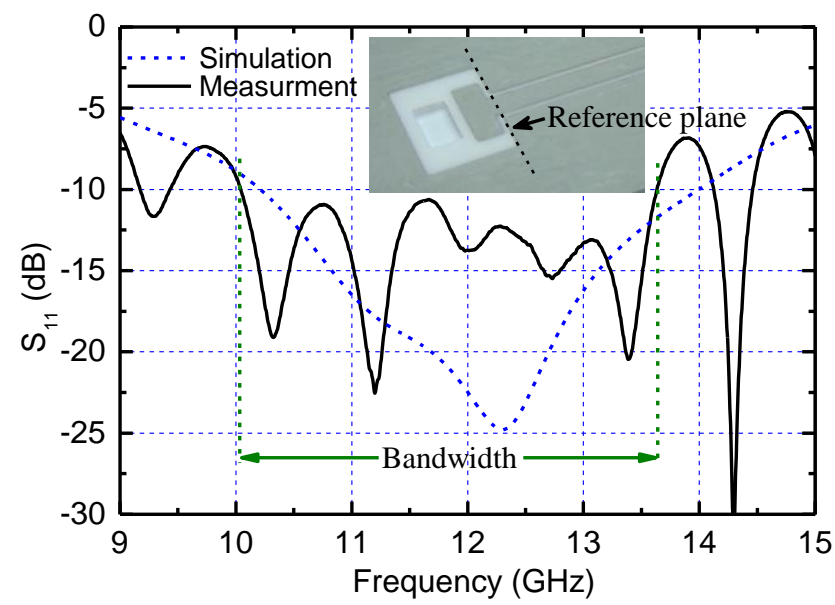

Fig. 14. The measured $S_{11}$ response of the interrogation antenna.

\section{Measurement setup and results}

A measurement setup is custom-made for the pressure measurement at high temperatures as shown in Fig. 15(a). A heat pad (Micropyretics Heaters International Inc.) is used to control the temperature of the pressure sensor under test with feedback from a $K$-type thermocouple (Omega HH11). A force is applied on the pressure sensor by using an alumina rod. In addition, a force gauge (Dillon GL050), attached on a test stand (Dillon CT), is used to provide precise control of applied force on the pressure sensor. Alumina sheets and alumina wool are covered on the heat pad to stabilize the temperature. Fig. 15(b) illustrates the detailed view of the pressure sensor under test. The alumina rod passes through the open window on the antenna substrate. Therefore, a force is applied via the alumina rod on the pressure sensor.

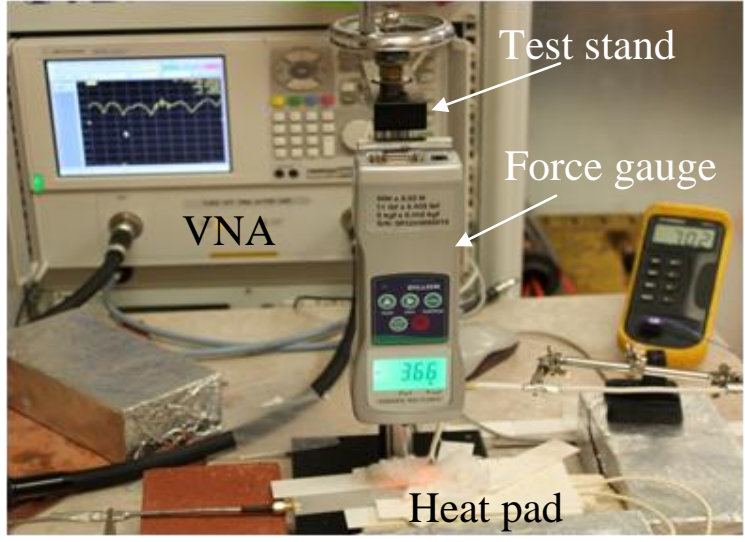

(a)

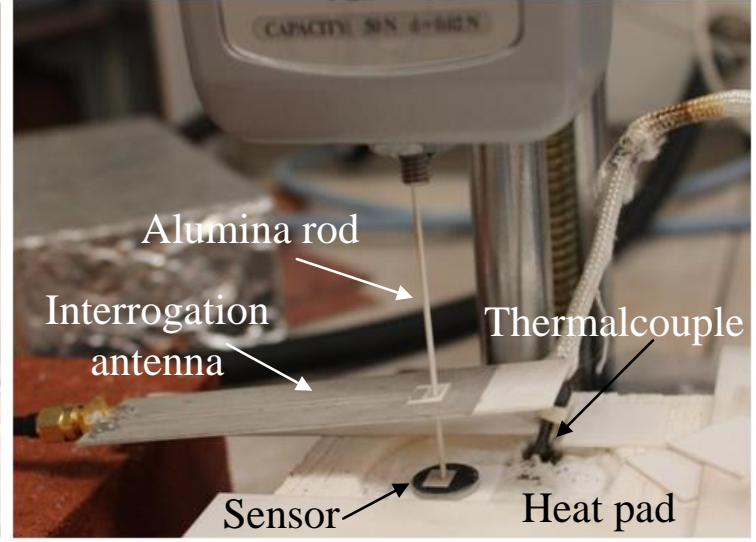

(b)

Fig. 15. The measurement setup. (a) Pressure sensor measurement under high temperatures and external forces. (b) The external force is applied on the pressure sensor which is below the interrogation antenna.

Using this measurement setup, the pressure and temperature can be independently and simultaneously applied to the pressure sensor with precise control. Therefore, the change of the pressure sensor $f_{r}$ due to the deflection of the sensor cap can be characterized at each temperature and applied force. This real-time measurement is performed with the Agilent 40-GHz PNA-L after time-domain gating [28].

The pressure sensor is measured at different temperatures up to $800^{\circ} \mathrm{C}$ with various forces up to $5 \mathrm{~N}$. The measured resonant frequency $f_{r}$ is plotted in Fig. 16(a) for different temperatures and forces. It is observed that the $f_{r}$ without applied force at room temperature is $12.2 \mathrm{GHz}$ which is close to the simulated frequency of $11.9 \mathrm{GHz}$. In addition, the resonant frequency $f_{r}$ decreases with the increasing applied force in a quasi-linear fashion due to reduced gap dimension. For example, the resonant frequency is reduced from 11.75 to $11.56 \mathrm{GHz}$ when the external force increases from 0 to $5 \mathrm{Newton}$ at $800^{\circ} \mathrm{C}$. It should be noted that the resonant frequency $f_{r}$ is also affected by the temperature as shown in Fig. 16(a). This can be caused by multiple physics mechanisms such as cavity deformation due to material expansion, different thermal expansion coefficients in the cap and 
bottom structures, residual stress inside PDC and metals. HFSS simulations reveal that the dielectric constant change from 25 to $800^{\circ} \mathrm{C}$ contributes approximately $10 \%$ of the overall frequency shift. Therefore, a temperature sensor is necessary for pressure sensor application to calibrate the frequency drift due to the temperature effect. The $\mathrm{Q}$ factor of the pressure sensor under 5-N loading decreases from 84.4 to 41.4 when the temperature increases from 25 to $800^{\circ} \mathrm{C}$. The maximum wireless sensing range between the interrogation antenna and the pressure sensor depends on both temperature and pressure of the sensor, which correspond to different $\mathrm{Q}$ factors. The maximum sensing range is approximately $15 \mathrm{~mm}$. This range is reduced when the temperature and pressure are raised. It is noted that a network analyzer (with limited power and dynamic range) is used for the measurement. The wireless sensing range is $10 \mathrm{~mm}$ in order to obtain strong resonance peaks from the sensor. This sensing range could be significantly increased by using customized amplification circuits. The sensor response above $800^{\circ} \mathrm{C}$ is not detectable, possibly due to the increased metallic loss of the platinum layer and dielectric loss of the PDC substrate. During the measurement when the applied force was above $10 \mathrm{~N}$, cracks were observed inside the sensor cap. Therefore, pressure sensor measurements were conducted for external force between 0 and $5 \mathrm{~N}$ to collect repeatable responses.

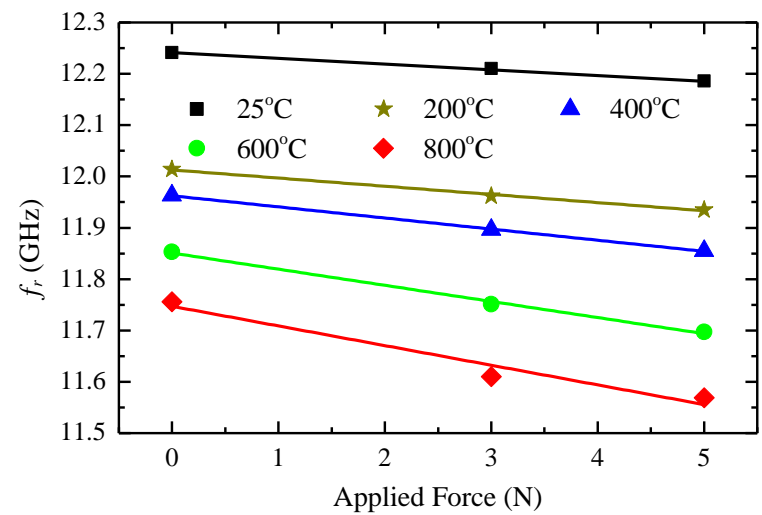

(a)

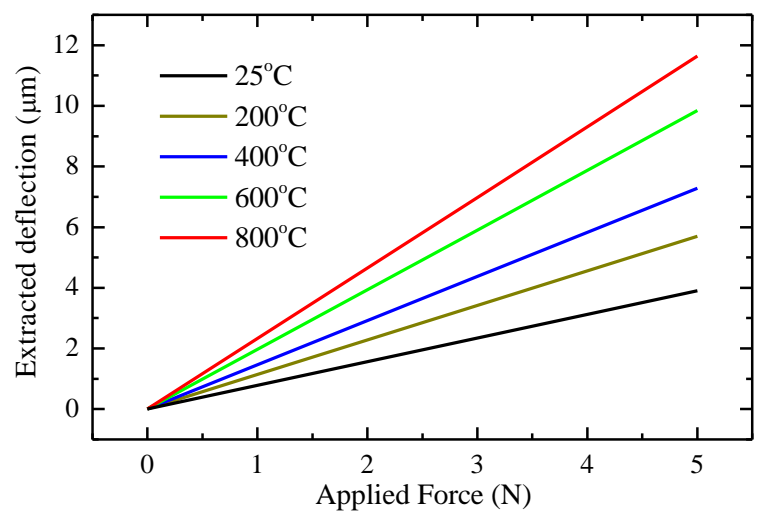

(b)

Fig. 16. (a) Measured resonant frequency $f_{r}$ of the pressure sensor versus the applied force at each temperature. (b) Extracted deflection of the pressure sensor cap due to the applied force at different tempertures.

By combining the sensitivity analysis in Fig. 4(b) and the $f_{r}$ change in Fig. 16(a), the sensor cap deflection versus applied force at different temperatures are calculated and plotted in Fig. 16(b). The maximal deflection of $11.6 \mu \mathrm{m}$ due to the external force of $5 \mathrm{~N}$ is much smaller than the designed $69.8 \mu \mathrm{m}$ as shown in Fig. 5(a). In addition, Fig. 16(b) shows that at higher temperatures, larger deflection occurs for the same amount of change in the applied force, which implies that the sensor structure becomes less stiff. This can be due to the change in the Young's modulus of PDC and platinum layer. In addition, the aforementioned cavity deformation can also affect this sensitivity change.

Several pressure sensors for harsh-environment applications are summarized in Table 3. It can be observed that much higher sensor sensitivity is achieved by using the evanescent-mode resonator, compared with other pressure sensors based on $L C$ resonators. In addition, the sensor proposed in this paper is able to work up to $800^{\circ} \mathrm{C}$ due to the high $\mathrm{Q}$ factors. It should be noted that the sensor deformation in this paper is obtained with an external force instead of pressure.

Table 3. Characteristics of different pressure sensors for harsh-environment applications.

\begin{tabular}{|c|c|c|c|c|c|}
\hline & Working mechanism & Sensor materials & $\begin{array}{l}\text { Highest working } \\
\text { temperature }\left({ }^{\circ} \mathrm{C}\right)\end{array}$ & $\begin{array}{l}\text { Highest working } \\
\text { pressure (psi) }\end{array}$ & $\begin{array}{l}\text { Sensitivity } \\
\text { (MHz/psi) }\end{array}$ \\
\hline [9-11] & $L C$ resonator & $\begin{array}{l}\text { Low-temperature co-fired } \\
\text { ceramic and silver }\end{array}$ & 400 & 101.5 & 0.0097 \\
\hline [9-11] & $L C$ resonator & $\begin{array}{l}\text { Low-temperature co-fired } \\
\text { ceramic and silver }\end{array}$ & 600 & 52.2 & 0.024 \\
\hline [9-11] & $L C$ resonator & $\begin{array}{l}\text { High-temperature co-fired } \\
\text { ceramics and platinum }\end{array}$ & 600 & 43.5 & 0.059 \\
\hline $\begin{array}{l}\text { This } \\
\text { work }\end{array}$ & $\begin{array}{l}\text { Evanescent-mode } \\
\text { resonator }\end{array}$ & PDC and platinum & 800 & $77.9^{*}$ & 2.44 \\
\hline
\end{tabular}

*It is noted that the pressure applied to the sensor is through a dielectric rod not ambient gas. Therefore, the actual highest working pressure under ambient gas could be different. 


\section{Conclusion and discussion}

In this paper, we successfully demonstrated a wireless passive pressure sensor up to $800^{\circ} \mathrm{C}$, owing to the robust PDC material and high-Q evanescent-mode resonator structure. This pressure sensor is compact by using an integrated patch antenna. In addition, further studies will be conducted in PDC material synthesis to reduce loss at high temperatures, thereby increasing the work temperatures of the pressure sensor above $1000^{\circ} \mathrm{C}$. Integrating a wireless temperature sensor [33] with the pressure sensor will calibrate the frequency shift. A wireless sensor test setup with ambient high temperatures and high pressures will be constructed to measure these pressure sensors.

\section{Acknowledgment}

The authors acknowledge the support from US Department of Energy under Grant DE-FE0001241.

\section{References}

[1] M. P. Boyce, Gas Turbine Engineering Handbook, Third Edition: Gulf Professional Publishing, 2006.

[2] P. Herfurth et al. , "Ultrathin Body InAIN/GaN HEMTs for High-Temperature $\left(600^{\circ} \mathrm{C}\right)$ Electronics," IEEE Electron Device Letters, vol. 34, no. 4, pp. 496-498, 2013.

[3] J. Yang, "A Harsh Environment Wireless Pressure Sensing Solution Utilizing High Temperature Electronics," Sensors, vol. 13, no. 3, pp. 2719-2734, 2013.

[4] S. Guo, H. Eriksen, K. Childress, A. Fink, and M. Hoffman, "High temperature smart-cut SOI pressure sensor," Sensors and Actuators A: Physical, vol. 154, no. 2, pp. 255-260, 2009.

[5] S. Fricke, A. Friedberger, H. Seidel, and U. Schmid, "A robust pressure sensor for harsh environmental applications," Sensors and Actuators A: Physical, vol. 184, pp. 16-21, 2012.

[6] D. J. Young, J. Du, C. A. Zorman, and W. H. Ko, "High-temperature single-crystal 3C-SiC capacitive pressure sensor," IEEE Sensors Journal, vol. 4, no. 4, pp. 464-470, 2004.

[7] Q. Tan et al. , "A High Temperature Capacitive Pressure Sensor Based on Alumina Ceramic for in Situ Measurement at $600^{\circ}$ C," Sensors, vol. 14, no. 2, pp. 2417-2430, 2014.

[8] H. A. Walter, H. Honen, and H. E. Gallus, "Adaptor for monitoring a pressure sensor to a gas turbine housing," US5612497 A, 1997.

[9] M. A. Fonseca, J. M. English, M. Von Arx, and M. G. Allen, "Wireless micromachined ceramic pressure sensor for high-temperature applications," Journal of Microelectromechanical Systems, vol. 11, no. 4, pp. 337-343, 2002.

[10] J. Xiong et al. , "Wireless LTCC-based Capacitive Pressure Sensor for Harsh Environment," Sensors and Actuators A: Physical, vol. 197, pp. 30-37, 2013.

[11] Q. Tan et al. , "A Wireless Passive Pressure Microsensor Fabricated in HTCC MEMS Technology for Harsh Environments," Sensors, vol. 13, no. 8, pp. 9896-9908, 2013.

[12] D. Senior et al., "Wireless passive sensing application using a cavity loaded evanescent mode half mode substrate integrated waveguide resonator," presented at the 16th International TRANSDUCERS conference, Beijing, China, 2011.

[13] Y. Zhao et al. , "RF evanescent-mode cavity resonator for passive wireless sensor applications," Sensors and Actuators A: Physical, vol. 161, no. 1, pp. 322-328, 2010.

[14] A. Mahmood, H. H. Sigmarsson, H. Joshi, W. J. Chappell, and D. Peroulis, "An evanescent-mode cavity resonator based thermal sensor," presented at the IEEE Sensors, Atlanta, GA, 2007.

[15] H. Cheng, S. Ebadi, and X. Gong, "A wireless pressure sensor design using a microwave cavity resonator," presented at the IEEE AP-S International Symposium, Chicago, IL, 2012.

[16] Y. Wang et al. , "A silicoaluminum carbonitride ceramic resist oxidation/corrosion in water vapour," Journal of Materials Research, vol. 21, no. 7, pp. 1625-1628, 2006.

[17] N. Ravi Kumar et al., "Crystallization and creep behavior of Si-B-C-N ceramics," Acta materialia, vol. 53, no. 17, pp. 4567-4578, 2005.

[18] X. Ren, S. Ebadi, Y. Chen, A. Linan, and X. Gong, "Characterization of SiCN Ceramic Material Dielectric Properties at High Temperatures for Harsh Environment Sensing Applications," IEEE Transactions on Microwave Theory and Techniques, vol. 61, no. 2, pp. 960-971, 2013.

[19] X. Gong et al. , "Precision fabrication techniques and analysis on high-Q evanescent-mode resonators and filters of different geometries," IEEE Transactions on Microwave Theory and Techniques, vol. 52, no. 11, pp. 2557-2566, 2004.

[20] X. Gong, W. J. Chappell, and L. Katehi, "Reduced size capacitive defect EBG resonators," presented at the IEEE MTTS International Microwave Symposium Digest, Seattle, WA, 2002.

[21] H. Joshi, H. H. Sigmarsson, D. Peroulis, and W. J. Chappell, "Highly loaded evanescent cavities for widely tunable high-Q filters," presented at the IEEE MTT-S International Microwave Symposium Digest, Honolulu, HI, 2007. 
[22] M. S. Arif and D. Peroulis, "All-Silicon Technology for High-Q Evanescent Mode Cavity Tunable Resonators and Filters," Journal of Microelectromechanical Systems, no. 99, 2013.

[23] S.-J. Park, I. Reines, C. Patel, and G. M. Rebeiz, "High-RF-MEMS 4-6-GHz tunable evanescent-mode cavity filter," IEEE Transactions on Microwave Theory and Techniques, vol. 58, no. 2, pp. 381-389, 2010.

[24] S. Hajela, X. Gong, and W. J. Chappell, "Widely tunable high-Q evanescent-mode resonators using flexible polymer substrates," presented at the IEEE MTT-S International Microwave Symposium Digest, 2005.

[25] Y. Tian, G. Shao, X. Wang, and L. An, "Fabrication of nano-scaled polymer-derived SiAlCN ceramic components using focused ion beam," Journal of Micromechanics and Microengineering, vol. 23, no. 9, p. 095035, 2013.

[26] X. Gong, A. Margomenos, B. Liu, W. J. Chappell, and L. P. Katehi, "High-Q evanescent-mode filters using silicon micromachining and polymer stereolithography $(S L)$ processing," in IEEE MTT-S International Microwave Symposium Digest, 2004, pp. 433-436.

[27] Y. Yusuf, C. Haitao, and G. Xun, "Co-designed substrate-integrated waveguide filters with patch antennas," IET Microwaves, Antennas \& Propagation, vol. 7, no. 7, pp. 493-501, 2013.

[28] H. Cheng, S. Ebadi, X. Ren, Y. Yusuf, and X. Gong, "A compact wireless passive sensing mechanism based on a seamlessly integrated resonator/antenna," presented at the IEEE AP-S International Symposium, Spokane, WA, 2011.

[29] H. Cheng, X. Ren, S. Ebadi, and X. Gong, "A Wide-band Square Slot Antenna for High-Temperature Applications," presented at the IEEE AP-S International Symposium, Orlando, FL, 2013.

[30] D. Seo et al. , "Fabrication and electrical properties of polymer-derived ceramic (PDC) thin films for high-temperature heat flux sensors," Sensors and Actuators A: Physical, vol. 165, no. 2, pp. 250-255, 2011.

[31] A. Leo, S. Andronenko, I. Stiharu, and R. B. Bhat, "Characterization of thick and thin film SiCN for pressure sensing at high temperatures," Sensors, vol. 10, no. 2, pp. 1338-1354, 2010.

[32] J. Liu, J. Li, and C. Xu, "Cutting Force Prediction on Micromilling Magnesium Metal Matrix Composites With Nanoreinforcements," ASME Transaction, Journal of Micro and Nano-Manufacturing, vol. 1, no. 1, pp. 011010-1 011010-10, 2013.

[33] H. Cheng, S. Ebadi, and X. Gong, "A Low-Profile Wireless Passive Temperature Sensor Using Resonator/Antenna Integration Up to $1000^{\circ} \mathrm{C}$," IEEE Antennas and Wireless Propagation Letters, vol. 11, pp. 369-372, 2012.

[34] "Agilent network analysis applying the 8510 TRL calibration for non-Coaxial measurements," Agilent Technologies Corp., Santa Clara, CA, Product note 8510-8A 2001. 